\title{
medios auxiliares para la carga de traillas
}

$863 \cdot 24$

\section{sinopsis}

La disparidad entre las potencias necesarias para efectuar la carga de las traíllas y la utilizable en el transporte del material influyen poderosamente en el rendimiento económico del transporte de tierras mediante este tipo de maquinaria.

Esto ha exigido el estudiar y desarrollar diversos sistemas que permitan el empleo de máquinas dotadas de motores de menor potencia $y$, consiguientemente, de menor consumo y coste inicial.

Entre los sistemas comúnmente empleados para facilitar las operaciones de carga figuran el empleo de tractores auxiliares empujadores, el auxilio mutuo de traíllas y máquinas dotadas de sistemas mecánicos que eviten la penetración del material a través del previamente introducido.

El empleo de tractores auxiliares empujadores implica numerosas servidumbres, además de una mayor capitalización de la maquinaria necesaria para realizar el trabajo.

Las traillas dotadas de sistemas mecánicos elevadores de la carga necesitan una potencia de arrastre para efectuar tal operación mucho menor y, por lo tanto, el promedio de potencia disponible y potencia utilizada durante el ciclo es más favorísticas que impiden que esta solución sea la óptima para toda clase de trabajos.

Pese al constante incremento de aplicación de procedimientos vibratorios en maquinaria no se ha generalizado el empleo de la vibración en la operación de carga en las traíllas, sin duda debido a los volúmenes y pesos implicados en el problema.

Siempre que el trabajo no se esté efectuando con una unidad sobredimensionada, o el material sea excepcionalmente dócil, será preciso disponer de un medio auxiliar de carga que facilite dicha operación e incremente el rendimiento, al disminuir el tiempo necesario para realizarla y aumentar el volumen de la misma (ver núm. 135 de Informes).

Pueden considerarse como medios auxiliares, en la carga de traíllas, las unidades motrices empujadoras y los dispositivos mecánicos de que están dotados algunos modelos y que tienen por misión evitar la resistencia que encuentra el material que está introduciéndose en la caja al tener que atravesar la carga introducida previamente.

\section{Unidades empujadoras}

Pueden ser consideradas como tales: los motores auxiliares, los tractores empujadores y las traíllas que trabajan en ayuda mutua.

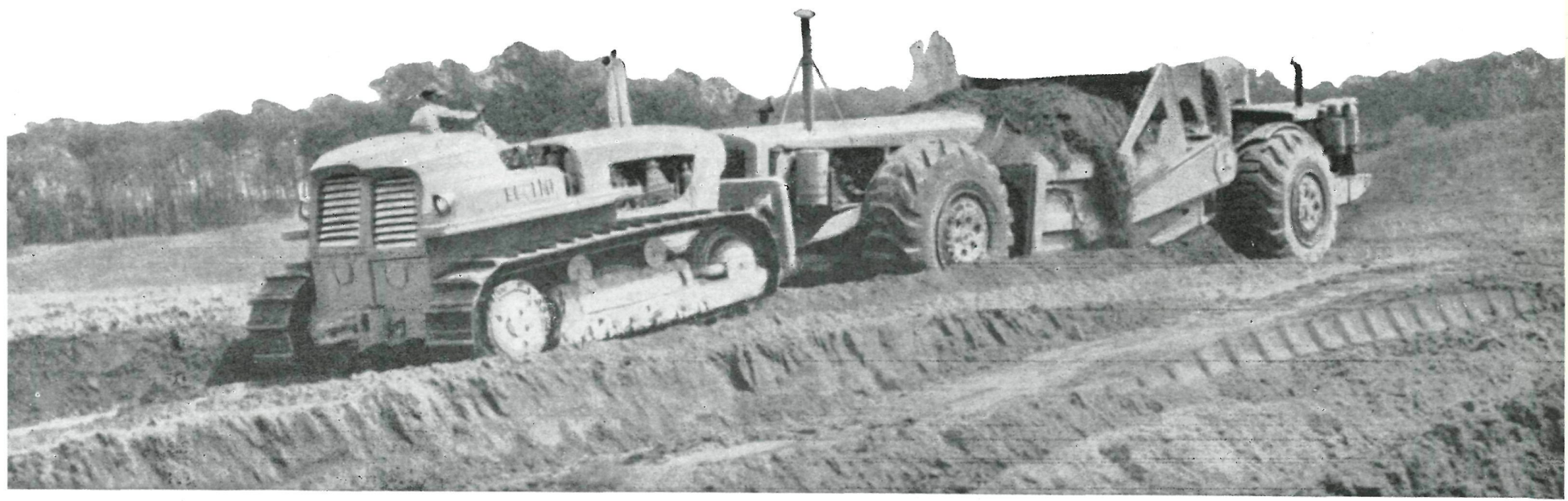


Las traíllas dotadas de motor suplementario están proyectadas, más que con el fin de disponer de la potencia indispensable para efectuar la carga, con objeto de poder utilizar, durante el transporie del material, unidades motrices menores que proporcionen la potencia suficiente para efectuar el mismo, sin incrementar, no obstante, innecesariamente el coste por unidad de carga transportada.

Los tractores empujadores empleados para auxilio de la operación de carga de las traíllas han sido, generalmente, tractores de orugas, hasta que los perfeccionamientos obtenidos en los neumáticos de baja presión han permitido el empleo económico de los mismos en la ejecución de estas operaciones. La elección del tipo de tractor auxiliar a emplear ha de basarse en las disponibilidades existentes y en las características del trabajo a desarrollar, pero, pese a reunir las condiciones adecuadas, las servidumbres de la operación de auxilio por el tractor empujador son grandes y numerosas.

Normalmente, y con el fin de poder ser empleados en trabajos de nivelación cuando la labor lo permita, estos tractores irán dotados de cuchilla empujadora, lo que incrementará la dificultad de la maniobra de empuje, ya que, al desplazarse las máquinas en planos diferentes por las irregularidades del terreno, se aumentarán las dificultades inherentes a la maniobra de propulsión. Asimismo, la potencia desarrollada por el tractor empujador estará subordinada a la pericia de los operarios, con objeto de evitar que ambas unidades no trabajen en el mismo eje de marcha. Será inevitable también, pese al cuidadoso estudio y planteamiento de los ciclos y de los equipos, que el equilibrio de éstos será alterado y, por lo tanto, habrá que recurrir a soluciones de emergencia, tales como tener que efectuar media carga sin auxilio del empujador, para evitar maquinaria inmovilizada.

Las ventajas obtenidas mediante el empleo de tractores auxiliares para la carga son, sin embargo, de excepcional importancia.

El peso de la carga puede considerarse incrementado de un 30 a un $40 \%$; el tiempo necesario para efectuar la misma, reducido a un $50 \%$; y la longitud necesaria para efectuarla, acortada en un $40 \%$.

El siguiente gráfico indica los incrementos obtenidos mediante el empleo de tractor auxiliar de empuje en la carga transportada por una traílla, en función de la carga práctica de la misma sin empujador y la duración del ciclo en minutos.

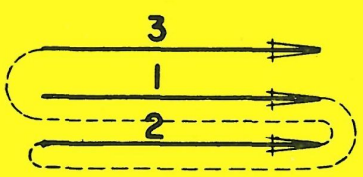

EMPUJE NORMAL

Trabajo en tramos cortos en dirección única.

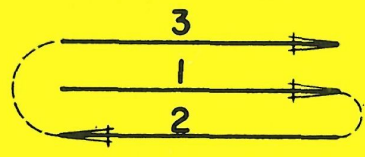

EMPUJE EN VAIVEN

Trabajo en tramos cortos en dos direcciones.

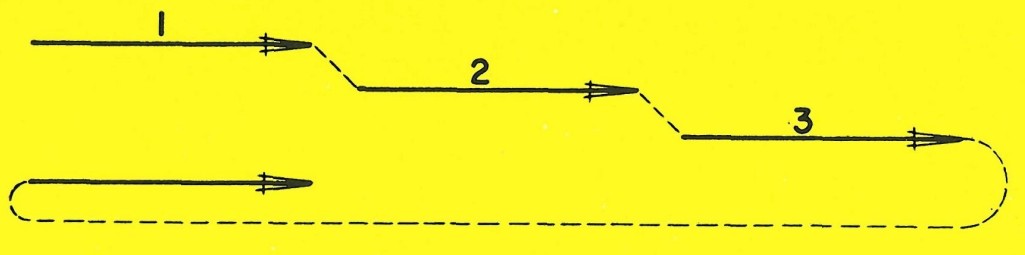

EMPUJE EN GADENA

Utilizable excepcionalmente en tramos la:gos. 
El empleo del sistema de auxilio mutuo de traíllas puede considerarse como un caso particular dentro del auxilio por tractor empujador, con la servidumbre del mayor peso muerto del elemento empujador y su menor maniobrabilidad, y la ventaja de economizar elementos al aprovechar, para labores auxiliares, maquinaria que tenemos movilizada para el trabajo.

La complejidad que requieren los sistemas de auxilio para la carga de traíllas, así como la mayor capitalización de la maquinaria necesaria, unido a un aprovechamiento medio de la potencia imprescindible para la carga sumamente baja con el siguiente encarecimiento, han inducido a la realización de cajas, de diseño adecuado, que tiendan a evitar el efecto «punching», y a proyectar sistemas mecánicos de carga que realizan la misma evitando introducir en la caja el material a través del previamente cargado.

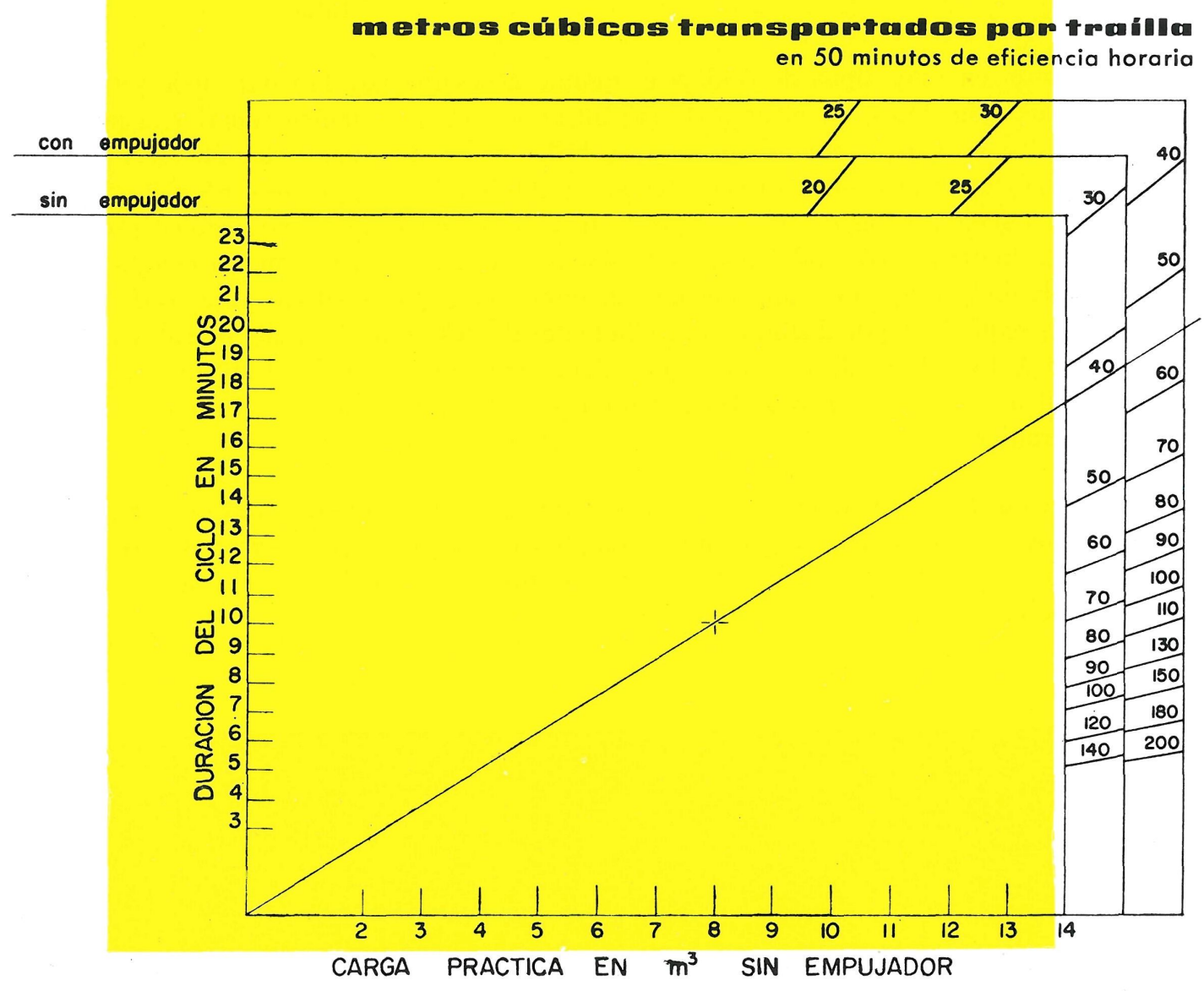

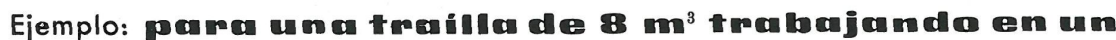
cíclo de 10 minutros, 40 me $^{3}$ sin empujador y 5

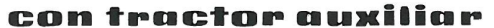

\title{
Mecanismos cargandores
}

Como ejemplo típico se pueden citar las traíllas dotadas de mecanismos cargadores que, accionados, bien por motores independientes, bien por medio de la barra del tractor, efectúan la carga del material arrancado por el borde de la cuchilla depositando el mismo sobre el introducido en la caja con anterioridad. Efectivamente, la potencia necesaria para realizar la carga en estos tipos de traíllas es mucho menor que en las traíllas convencionales. Para traíllas de $5 \mathrm{~m}^{3}$ de capacidad son suficientes tractores de 45 HP. y de $290 \mathrm{HP}$. para traíllas de 16 metros cúbicos. 


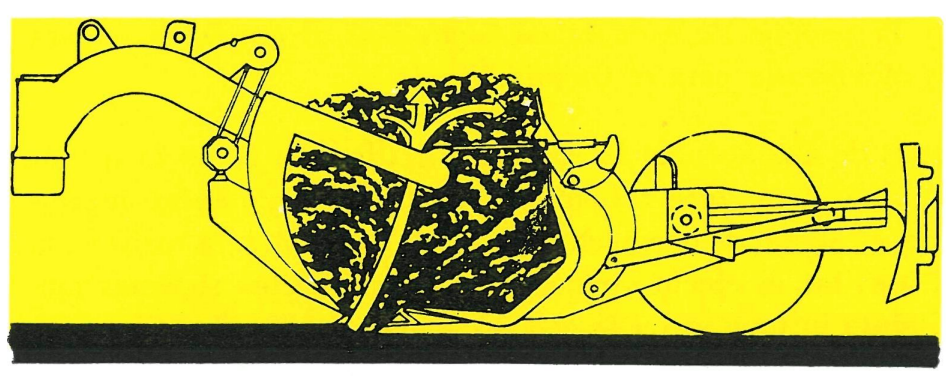

Estos sistemas presentan, por tanto, una ventaja indudable en cuanto a potencia necesaria para efectuar las cargas, lo que trae consigo un aprovechamiento óptimo de la potencia media empleada durante el ciclo al ser menos dispares la potencia imprescindible para la carga y la exigida para el transporte $y$, consecuentemente, un rendimiento económico más favorable.

Sin embargo, en estos tipos de traíllas el tiempo necesario para la carga suele ser mayor, ya que los dispositivos cargadores, en el caso de encontrar sólidos de volumen superior al permitido por la separación entre las paletas cargadoras y la cuchilla, se desembragan automáticamente y sufren, por tanto, interrupciones más o menos frecuentes en la continuidad del proceso. El efecto de deslizamiento del material suele ser mayor en este tipo de traíllas, así como también el polvo producido, por lo que parte del material arrancado no es introducido en la caja. Asimismo, la complejidad mecánica imprescindible en este tipo de traíllas es mayor, tanto en lo que afecta al proceso de carga como en las exigencias requeridas por distintos procedimientos de descarga. Por todo lo cual, y aun teniendo en cuenta que este tipo de traíllas es muy conveniente para determinados trabajos, no puede considerarse este sistema como el único indicado para resolver el problema de las potencias necesarias para la carga de traíllas.

La aplicación de procedimientos vibratorios para facilitar las operaciones de carga de las traíllas no parece, pese a su continuo incremento de empleo en maquinaria, haberse generalizado ni haber obtenido una adaptación específica para la carga de traíllas, sin duda debido a los volúmenes y pesos implicados en el problema.

M. CHINCHILLA

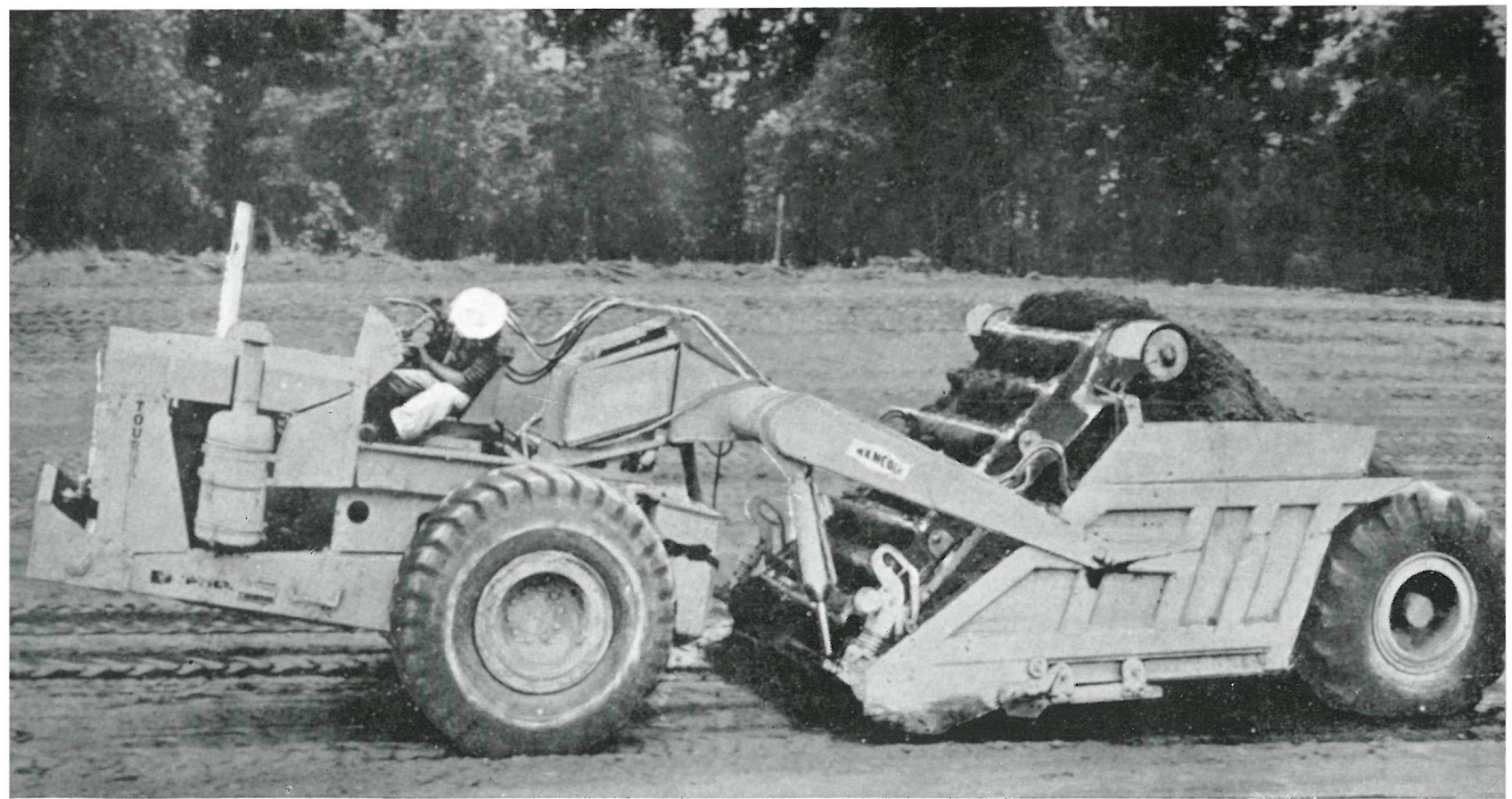




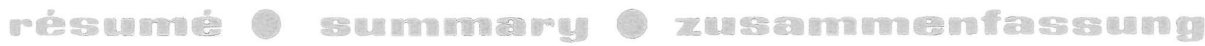

\section{Moyems auxiliaines poum la change de scrupers}

La différence entre les puissances nécessaires pour effectuer la charge des scrapers et celle qu'on utilise pour le transport des matériaux influe considérablement sur le rendement économique du transport de terrassements au moyen de ce type d'engins.

Pour cette raison, on a dû étudier et développer plusieurs systèmes qui permettent l'emploi de machines dotées de moteurs moins puissants et, par conséquent, de moindre consommation et de prix de revient initiel moins élevé.

Parmi les systèmes employés le plus couramment pour faciliter les opérations de charge figurent l'emploi de tracteurs auxiliaires pousseurs, l'aide mutuelle des scrapers et engins dotés de systèmes mécaniques qui évitent la pénétration du matériau à travers celui introduit préalablement.

L'emploi de tracteurs auxiliaires pousseurs demande le concours d'un personnel nombreux, en plus d'une plus forte capitalisation de l'équipement nécessaire pour l'exécution du travail.

Les scrapers dotés de systèmes mécaniques élévateurs de charge nécessitent une puissance de trait pour effec. tuer ce travail beaucoup moins important et, par conséquent, la moyenne de puissance disponible et la puis sance utilisée pendant le cycle sont plus favorables que pour les scrapers conventionnels. Cependant, leur emploi est également subordonné à plusieurs conditions et caractéristiques qui empêchent cette solution d'être l'idéale pour toute sorte de travaux.

Malgré l'augmentation constante d'application de procédés de vibration pour les machines, leur emploi pour les opérations de charge de scrapers ne s'est pas généralisé sans aucun doute, à cause des volumes et des poids impliqués dans le problème.

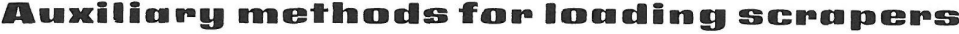

The large difference in the power that is necessary to load the scraper and to transport the material has an important influence on the economic efficiency involved in transporting soil with this type of equipment. This problem has made it necessary to develop various kinds of machinery fitted with less powerful engines, and hence, having a smaller fuel consumption and of lower initial cost.

Among the systems normally adopted to help the loading operation the following may be cited: auxiliary pushing tractors; mutual aid by several scrapers; and machinery fitted with mechanical means to avoid material from penetrating into other material that is already loaded.

The use of auxiliary pushing tractors requires many additional services, and also a greater capital outlay in equipment.

The scrapers fitted with a mechanical system for lifting the load need a much smaller drawing power to carry out this operation, and consequently the mean available and mean required power during the operating cycle is more advantageous than in ordinary scrapers. Even so, the utilisation of these new type scrapers is also subjected to various limiting circumstances which prevent this from being the best possible solution for this type of work.

In spite of the increasing use of vibratory techniques, so far vibration has not yet become a generalised procedure in loading of scrapers, no doubt due to the large volumen and mass of material involved in the procedure.

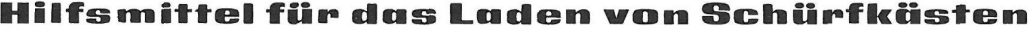

Die Ungleichheit zwischen den notwendigen Leistungen zur Durchführung des Ladens der Schürfkästen und die beim Materialtransport gebräuchliche beeinfiussen stark die wirtschaftliche Leistung der Beförderung von Erden mittels dieser Maschinenart.

Dies erforderte das Studium und die Entwicklung von verschiedenen Systemen, welche die Anwendung von Maschinen erlauben, die mit Motoren geringerer Leistung und infolgedessen mit geringerem Verbrauch und Anschaffungspreis versehen sind.

Unter den gewöhnlich angewandten Systemen zur Erleichterung der Tätigkeit des Ladens erscheinen die Anwendung von Hilfsschubtraktoren, die gegenseitige Hilfe von Schürfkästen und die mit mechanischen Systemen versehenen, die das Eindringen des Materiales durch das vorher eingeführte verhindern.

Die Anwendung von Hilfsschubtraktoren begreift zahlreiche Dienste ein, abgesehen von einer grösseren Kapitalisierung der zur Durchführung der Arbeit notwendigen Maschinen.

Die mit mechanischen Systemen zur Hebung der Lasten versehenen Schürfkästen benötigen eine weit geringere Geschiebe - Triebkraft, um eine derartige Tätigkeit durchzuführen, und deshalb ist der Durchschnitt der verfügbaren Kraft und genützten Leistung während des Kreislaufes günstiger als bei den herkömmlichen Schürfkästen. Jedoch ist ihre Anwendung auch verschiedenen Bedingungen und Kennzeichnungen unterstellt, die es verhindern, dass diese Lösung die beste für jede Art von Arbeiten ist.

Trotz der beständigen Zunahme der Anwendung von maschinellen Rüttlungsverfahren hat man die Verwen dung der Rüttlung bei dcr Tätigkeit des Ladens der Schürfkästen nicht verallgemeinert, zweifellos wegen der in das Problem verflochtenen Umfänge und Gewichte. 